\title{
Diverse Representations of Olfactory Information in Centrifugal Feedback Projections
}

\author{
Krishnan Padmanabhan, ${ }^{1,2,7}$ Fumitaka Osakada, ${ }^{3,6}$ Anna Tarabrina, ${ }^{4}$ Erin Kizer, ${ }^{4}$ Edward M. Callaway, ${ }^{1,3}$ \\ 달 H. Gage, ${ }^{4}$ and Terrence J. Sejnowski ${ }^{1,2,5}$ \\ ${ }^{1}$ Crick-Jacobs Center for Theoretical and Computational Biology, ${ }^{2}$ Computational Neurobiology Laboratory, ${ }^{3}$ Systems Neurobiology Laboratories, \\ ${ }^{4}$ Laboratory of Genetics, and ${ }^{5}$ Howard Hughes Medical Institute, Salk Institute for Biological Studies, La Jolla, California 92037, ${ }^{6}$ Laboratory of Cellular \\ Pharmacology, Graduate School of Pharmaceutical Sciences, Nagoya University, Nagoya, Aichi 464-8601, Japan, and ${ }^{7}$ Department of Neuroscience, \\ University of Rochester School of Medicine, Rochester, New York 14642
}

Although feedback or centrifugal projections from higher processing centers of the brain to peripheral regions have long been known to play essential functional roles, the anatomical organization of these connections remains largely unknown. Using a virus-based retrograde labeling strategy and 3D whole-brain reconstruction methods, we mapped the spatial organization of centrifugal projections from two olfactory cortical areas, the anterior olfactory nucleus $(\mathrm{AON})$ and the piriform cortex, to the granule cell layer of the main olfactory bulb in the mouse. Both regions are major recipients of information from the bulb and are the largest sources of feedback to the bulb, collectively constituting circuits essential for olfactory coding and olfactory behavior. We found that, although ipsilateral inputs from the AON were uniformly distributed, feedback from the contralateral AON had a strong ventral bias. In addition, we observed that centrifugally projecting neurons were spatially clustered in the piriform cortex, in contrast to the distributed feedforward axonal inputs that these cells receive from the principal neurons of the bulb. Therefore, information carried from the bulb to higher processing structures by anatomically stereotypic projections is likely relayed back to the bulb by organizationally distinct feedback projections that may reflect different coding strategies and therefore different functional roles.

Key words: centrifugal; circuit maps; feedback; olfaction; olfactory bulb; piriform cortex

\section{Significance Statement}

Principles of anatomical organization, sometimes instantiated as "maps" in the mammalian brain, have provided key insights into the structure and function of circuits in sensory systems. Generally, these characterizations focus on projections from early sensory processing areas to higher processing structures despite considerable evidence that feedback or centrifugal projections often constitute major conduits of information flow. Our results identify structure in the organization of centrifugal feedback projections to the olfactory bulb that is fundamentally different from the organization of feedforward circuits. Our study suggests that understanding computations performed in the olfactory bulb, and more generally in the olfactory system, requires understanding interactions between feedforward and feedback "maps" both structurally and functionally.

\section{Introduction}

Principles of anatomical organization often provide insight into how the sensory world is represented in patterns of neuronal activity. In the olfactory system of mammals, for example, the organization of feedforward projections to targets throughout the mouse brain

Received Sept. 2, 2015; revised June 1, 2016; accepted June 5, 2016.

Author contributions: K.P., E.M.C., F.H.G., and T.J.S. designed research; K.P., F.O., A.T., and E.K. performed research; K.P. and F.O. contributed unpublished reagents/analytic tools; K.P. analyzed data; K.P., E.M.C., F.H.G., and T.J.S. wrote the paper.

K.P. is supported by the National Institute of Mental Health (Grant K99 MH101634), a NARSAD Young Investigator Award, the Lieber Foundation, and a Crick-Jacobs Junior Fellowship. F.0. is supported by the Japan Society for the Promotion of Science, the Kanae Foundation for the Promotion of Medical Science, the Uehara Memorial Foundation, the Naito Foundation, the Takeda Science Foundation, and the Pioneer Fund. E.M.C. was supported by the National Institutes of Health and the Kavli Institute for Brain and Mind at University of California-San Diego. F.H.G.
(Mombaerts et al., 1996; Willhite et al., 2006; Soucy et al., 2009; Ghosh et al., 2011; Miyamichi et al., 2011; Sosulski et al., 2011) have

is supported by the Mathers Foundation, The JPB Foundation, and the Helmsley Foundation. T.J.S. is supported by Howard Hughes Medical Institute and the Office of Naval Research (Grants N000141310672 and N000141210299). We thank J. Fitzpatrick and J. Kasuboski for assistance with imaging; members of the Callaway, Gage, and Sejnowsk laboratories for providing helpful comments on this manuscript; and M.L. Gage and S. Skinner for feedback on the manuscript.

This article is freely available online through the J Neurosci Author Open Choice option.

Correspondence should be addressed to Krishnan Padmanabhan, Salk Institute, 10010 N. Torrey Pines Rd., La Jolla, CA 92037.E-mail: krishnan_padmanabhan@urmc.rochester.edu.

D0I:10.1523/JNEUROSCI.3358-15.2016

Copyright $\odot 2016$ Padmanabhan et al.

This is an Open Access article distributed under the terms of the Creative Commons Attribution License Creative Commons Attribution 4.0 International, which permits unrestricted use, distribution and reproduction in any medium provided that the original work is properly attributed. 
revealed principles of both olfactory coding (Davison and Ehlers, 2011; Babadi and Sompolinsky, 2014) and olfactory behavior (Kobayakawa et al., 2007; Root et al., 2014).

Axons from individual olfactory receptor neurons expressing one of $\sim 1000$ olfactory receptor genes in the olfactory epithelium project to one or two glomeruli in the main olfactory bulb (MOB) (Mombaerts et al., 1996). The location of glomeruli are highly stereotypic across both individuals and species (Soucy et al., 2009). This input convergence facilitates odor detection across many log orders of concentration (Meister and Bonhoeffer, 2001; Saito et al., 2009). Once olfactory information is processed in the bulb through local interactions with inhibitory neurons, spatially organized projections from the principal neurons (mitral/tufted cells, $\mathrm{M} / \mathrm{T}$ ) to downstream targets such as the anterior olfactory nucleus (AON) (Miyamichi et al., 2011) and the amygdala (Sosulski et al., 2011) drive innate behavioral responses (Kobayakawa et al., 2007; Root et al., 2014). In contrast, distributed projections from these same $\mathrm{M} / \mathrm{T}$ neurons to regions such as the piriform or olfactory cortex (Ghosh et al., 2011; Sosulski et al., 2011) are thought to be drivers of learned olfactory behaviors (Choi et al., 2011). Such anatomical differences highlight the varied roles that a single population of neurons can play based on the identity of their postsynaptic targets and the organization of projections to those targets.

However, such a feedforward model, in which each processing area successively assembles complex features into a unified percept of the stimulus to eventually drive behavior, belies the complexity of neuronal circuits. All sensory regions, including those in the olfactory system, make connections back to early sensory processing areas through feedback, or centrifugal connections (Price and Powell, 1970; Ahmed et al., 1994; Boyd et al., 2012; Markopoulos et al., 2012). Despite the accepted importance of centrifugal projections in olfactory coding (Boyd et al., 2015), it remains largely unknown what types of cells project back to the bulb and what, if any, organizational principles govern their spatial arrangement. If, for instance, centrifugal projections recapitulate the organization of feedforward projections, then the olfactory information that differentially drives innate versus learned behaviors could be preserved along anatomically segregated feedforward and feedback streams. Alternatively, if the architecture of feedback projections complements or is organized in different ways than those of feedforward circuits, then odor information carried by feedforward projections could be integrated and reshaped by centrifugal inputs back to the bulb, thereby changing the role that sensory input plays in driving behavior.

Therefore, to characterize the anatomical organization of feedback inputs in the olfactory system, we studied the spatial arrangement of centrifugally projecting neurons from two major cortical olfactory areas, the AON and the piriform cortex to the granule cell layer (GCL) of the MOB. The GCL is thought to be essential for much of the local computations performed in the olfactory system (Urban and Sakmann, 2002) and has been shown to be the largest recipient of centrifugal input from higher brain regions (Shipley and Adamek, 1984). In addition, both the AON and the piriform cortex are thought to be essential for how odors are represented, playing key roles in shaping olfactory behavior.

We found that projections from both of these areas were highly structured. Centrifugal inputs to the GCL from the ipsilateral AON were uniformly distributed, but projections from contralateral AON showed a strong ventral bias. In addition, piriform cortical neurons next to one another projected to simi- lar regions of the GCL, resulting in spatially clustered maps of feedback. This clustered organization contrasted the random structure of feedforward inputs described previously (Ghosh et al., 2011). We found that the structure of centrifugal projections from these two cortical areas are organized in anatomically different ways from the structure of feedforward inputs to these areas.

\section{Materials and Methods}

Rabies virus. Green fluorescent protein (GFP), mCherry, or blue fluorescent protein (BFP) was cloned in pSAD $\Delta \mathrm{G}-\mathrm{F} 3$ as described previously (Wickersham et al., 2007; Osakada et al., 2011; Osakada and Callaway, 2013). SAD $\Delta$ G-GFP, SAD $\Delta$ G-mCherry, and SAD $\Delta$ G-BFP were recovered in B7GG cells with transfection with the corresponding genomic plasmid, pcDNA-SADB19N, pcDNA-SADB19P, pcDNA-SADB19L, and pcDNA-SADB19G. Viruses were amplified in B7GG cells in a humidified atmosphere of $3 \% \mathrm{CO}_{2}$ and $97 \%$ air at $35^{\circ} \mathrm{C}$ and concentrated by two rounds of ultracentrifuging. The concentrated rabies viruses were titrated in HEK293t cells. The titers of the rabies viruses used in the present study were $5.0 \times 10^{8}$ to $3.0 \times 10^{9}$ infectious units $/ \mathrm{ml}$. The viruses were stored at $-80^{\circ} \mathrm{C}$ until use.

Animals. All experiments were performed in accordance with the guidelines for care and use of animals at the Salk Institute for Biological Studies. Twenty C57BL/6 mice (male and female) aged 3-4 months were used. One or two injections were made per animal ( $n=16$ animals, $n=23$ injections total). GCL was targeted using a stereotaxic coordinates (Kopf Instruments). Borosilicate micropipettes were pulled on a Sutter Instruments P200 and 40-200 $\mathrm{nl}$ of virus was delivered to each bulb via pulsed injection from a picospritzer (Parker). Three different fluorescent reporters in the G-deleted rabies virus were used for the experiments and all three labeled neurons extensively, including labeling of dendritic processes.

Targeting was confirmed by mapping labeled neurons at the injection site into a coordinate space corresponding to the GCL of the bulb.

Histology. Three to $10 \mathrm{~d}$ after the virus was injected into the GCL, animals were killed and perfused with $4 \%$ paraformaldehype (PFA) and brains were extracted and transferred to a solution of $4 \%$ PFA $/ 30 \%$ sucrose (Padmanabhan et al., 2010). Coronal sections measuring $100 \mu \mathrm{m}$ in thickness were made of the mouse brain from the bulb to approximately bregma $-4 \mathrm{~mm}$, allowing us to characterize inputs from all regions throughout the mouse brain. In three of the animals, damage while extracting the brain resulted in incomplete reconstruction of some regions such as the hippocampus and occipital cortex. In two additional animals, tissue warped during perfusion. Therefore, although we were able to identify and quantify the number of neurons retrogradely labeled by the rabies virus, we could not use these animals in the remapping analysis.

Imaging and data management. Images were acquired with an Olympus VS110 slide scanner. Sixteen-bit scans of all coronal sections were done at three to five virtual $Z$-planes for each section and then collapsed into a single maximum-intensity projection and down-sampled for analysis.

Image dilation and correlation. Individual coronal sections were manually aligned and the alignment was validated by correlation analysis (alignment validation was performed using custom functions in MATLAB (The MathWorks). First, after automated thresholding (equation 1 in Padmanabhan et al., 2010), tissue boundaries for each section were determined using the Moore-Neighbor tracing algorithm (Gonzalez et al., 2004). Briefly, we maximized the correlation as follows:

$$
\operatorname{corr}(x, y(i))=\frac{\sum_{j=i}^{m} n_{\mathrm{j}} *(j-\bar{x})}{\sqrt{\left(\sum_{j}^{m}(j-\bar{x})^{2} * n_{\mathrm{j}}\right) *\left(\frac{\left(N_{\mathrm{m}}-N_{\mathrm{i}}\right) * N_{\mathrm{i}}}{N_{\mathrm{m}}}\right)}}
$$

where $x$ is the original image and the thresholded image $y(i)$ at the threshold value $(I-1)$ with $j$ as the bit depth of the image (e.g., between 0 and 
255 for an 8-bit image), $n_{\mathrm{j}}$ as the number of pixels with the value $j$, and $\bar{x}$ as the mean pixel value of the image. In addition, $N_{\mathrm{m}}$ was the total number of pixels in the image and $N_{\mathrm{i}}$ was the number of pixels above the threshold value $(I-1)$. We rewrite our correlation as $\operatorname{corr}(x, y(m))$ where $x$ is the original image and $y(i)$ is the image thresholded at a value of $i$. The optimal correlation is then:

$$
\arg \max t(m)=\operatorname{corr}(x, y(m))
$$

and this value was selected as the threshold for segmenting images.

Cell finding. For each fluorescent image section, the AON and piriform cortex were identified. Only labels within these identified areas was used for analysis. To identify neurons, we defined an image $\mathrm{X}$ as $x_{\mathrm{n}}$ corresponds to the nth pixel as follows:

$$
\boldsymbol{x}=x_{1}, x_{2}, x_{3}, \ldots x_{\mathrm{n}}
$$

We defined another vector $y$ where $y_{\mathrm{n}}$ corresponding to the nth pixel:

$$
\begin{gathered}
y=y_{1}, y_{2}, y_{3}, \ldots y_{\mathrm{n}} \\
\text { such that } \quad y_{\mathrm{n}}\left\{\begin{array}{lll}
=1 & \text { if } x_{\mathrm{n}}>i \\
=0 & \text { else } x_{\mathrm{n}} \leq i
\end{array}\right.
\end{gathered}
$$

where $I$ was a value selected manually for thresholding. Thresholded images were then run through a series of image-processing functions to identify retrogradely labeled neurons. First, nearest-neighbor pixel grouping was used to assign contiguous pixels into neurons and, then, groups of pixels were sorted based on morphological properties.

Data analysis. All analyses described herein, including tissue identification, were done with custom functions written in MATLAB. Unless otherwise noted, error bars are SDs.

AON remapping. The edges of the AON were defined manually and then transformed from a Euclidean coordinate space into a polar coordinate space. After this, individual neurons were mapped onto this circular representation $(\Theta)$ while preserving nearest neighbor distances (Miyamichi et al., 2011). The MATLAB Circular Statistics Toolbox was used for some analyses. After mapping onto a unit circle, a vector representation reflected the mean number of neurons in the polar space for each experiment in both the ipsilateral and contralateral AON. In this representation, laminar information within subdivisions of the $\mathrm{AON}$ was discarded so as not to skew the magnitude of the vector representation. A Raleigh test for nonuniformity was used to determine the significance of distributions that appeared biased to one subdivision of the AON versus another.

Piriform remapping. For each coronal section of the piriform cortex, an arc was drawn manually through the cell-dense layer (layer 2) of the olfactory cortex. Individual cells were projected onto the arc corresponding to layer 2. Each of these arcs and the remapped neurons were then transformed into a line and points on that line (by mathematically flattering the curvature of the sections). The transformation preserved nearest neighbor distances and created a compact representation of the spatial distribution of retrograde-labeled neurons that could be analyzed. The $2 \mathrm{D}$ representation also provided a space from which random distributions of retrograde neurons could be generated to assess clustering.

Clustering was determined by calculating the pairwise distances between all retrograde neurons in a 2D space. Resampling was performed using the bootstrap method ( $90 \%$ of the total neurons). For random distributions, the positions of model cells were randomly drawn from all possible positions in the piriform cortex for that experiment. Let $n$ define the combination of all $x, y$ positions possible for a neuron to occupy in the space for each piriform cortex. Then $R \subseteq N$ is then a randomly chosen subset of $n$ where the number of $R$ random cells is matched to the number of labeled neurons for each experiment. Because different experiments resulted in different numbers of neurons being retrogradely labeled (due to differences in injection size), the number of random neurons varied with each experiment.

Cluster analysis. To analyze the spatial organization of neurons in the piriform cortex, 2D density maps were first generated. Borders between patches were identified by thresholding the density maps (either greater than the mean or greater than the mean $+1 \mathrm{SD}$ ) and then drawing edges around the binary images. Because bin size used to generate the density map and the threshold values used to identify the boundaries between the patches both affect features of clusters, including their size, shape, and the number of cells within a cluster, a combinatorial search of the space of different bin sizes and thresholds was performed for each measure to ensure that the result observed was not an artifact of parameter choice.

\section{Results}

\section{Visualizing centrifugal inputs to the GCL of the MOB}

To uncover the organizational principles of feedback in the olfactory system, a g-deleted rabies virus expressing a fluorescent reporter was stereotactically injected into the GCL (Haberly and Price, 1978) of the MOB in adult male and female mice (3-6 months of age; Fig. 1). Retrogradely labeled cells infected with the virus did not express the rabies glycoprotein, so fluorescent label was confined only to neurons at the injection site (Fig. $1 B$ ) and cells with axonal boutons that terminated at the site of the injection (Wickersham et al., 2007; Osakada and Callaway, 2013; Callaway and Luo, 2015). Because the organization of centrifugal connections to the glomerular layer (Petzold et al., 2009) and the mitral cell layer (Yan et al., 2008; Markopoulos et al., 2012) have been explored previously, we focused our analysis on feedback to the GCL. Injections varied in size from small injections confined to discrete regions of the GCL to larger injections throughout the GCL (Fig. 1C). The centers of injection locations were mapped by determining the center density of all labeled neurons in the bulb in a normalized coordinate space and this too varied by experiment (Fig. $1 C$, insert). After $3-10 \mathrm{~d}$ of viral infection $(n=6$ injections at $3 \mathrm{~d}, n=17$ injections at 5-10 d), animals were killed and coronal sections of the whole mouse brain were made. Across multiple sections (Fig. 1D), centrifugally projecting neurons were retrogradely labeled in the two major cortical olfactory processing areas, the ipsilateral and contralateral AON (Fig. 1D,E) and the piriform cortex (Fig. $1 F, G$ ). In addition, we found retrogradely labeled neurons in a number of brain areas including the nucleus of the lateral olfactory tract (nLOT), the horizontal diagonal band (HDB), amygdala regions, zona inserta, the piriformentorhinal cortex transition, and in various nuclei of the hypothalamus consistent with previous reports in other species (Shipley and Adamek, 1984).

\section{$3 \mathrm{D}$ reconstruction of centrifugal projections to the $\mathrm{MOB}$}

To further understand the spatial organization of centrifugal projections to the GCL of the MOB, we established a whole-brain imaging and analysis method for dissecting the structure of neuronal circuits (Fig. 2). First, fixed mouse brains were sliced in the coronal plane, generating datasets of $\sim 160-180$ sections $(100 \mu \mathrm{m})$. Individual sections were scanned with an automated fluorescence slide scanner, requiring $\sim 10-20 \mathrm{~h}$ per brain. Sections were aligned manually and this alignment was validated by measuring the correlation coefficient of adjacent sections. Finally, fluorescently labeled neurons were identified using automated methods and mapped into the $3 \mathrm{D}$ representation of the mouse brain for analysis (Padmanabhan et al., 2010).

In the example of a coronal section of the MOB in Figure $1 \mathrm{~A}$ (tissue edge $=$ light gray), a small injection was made to the dorsal GCL (dark gray). The algorithm identified neurons in the injection site (Fig. $1 B$, red points), as well as at the boundaries of the tissue (Fig. $1 B$, light gray). The boundary between the inner plexiform layer and the GCL (Fig. $2 B$, dark gray) was identified manually in each section. A 3D representation of the MOB (Fig. 2C) revealed the extent to which neurons were labeled throughout the bulb (Fig. $2 C$, red points). To quantify properties of the injection 

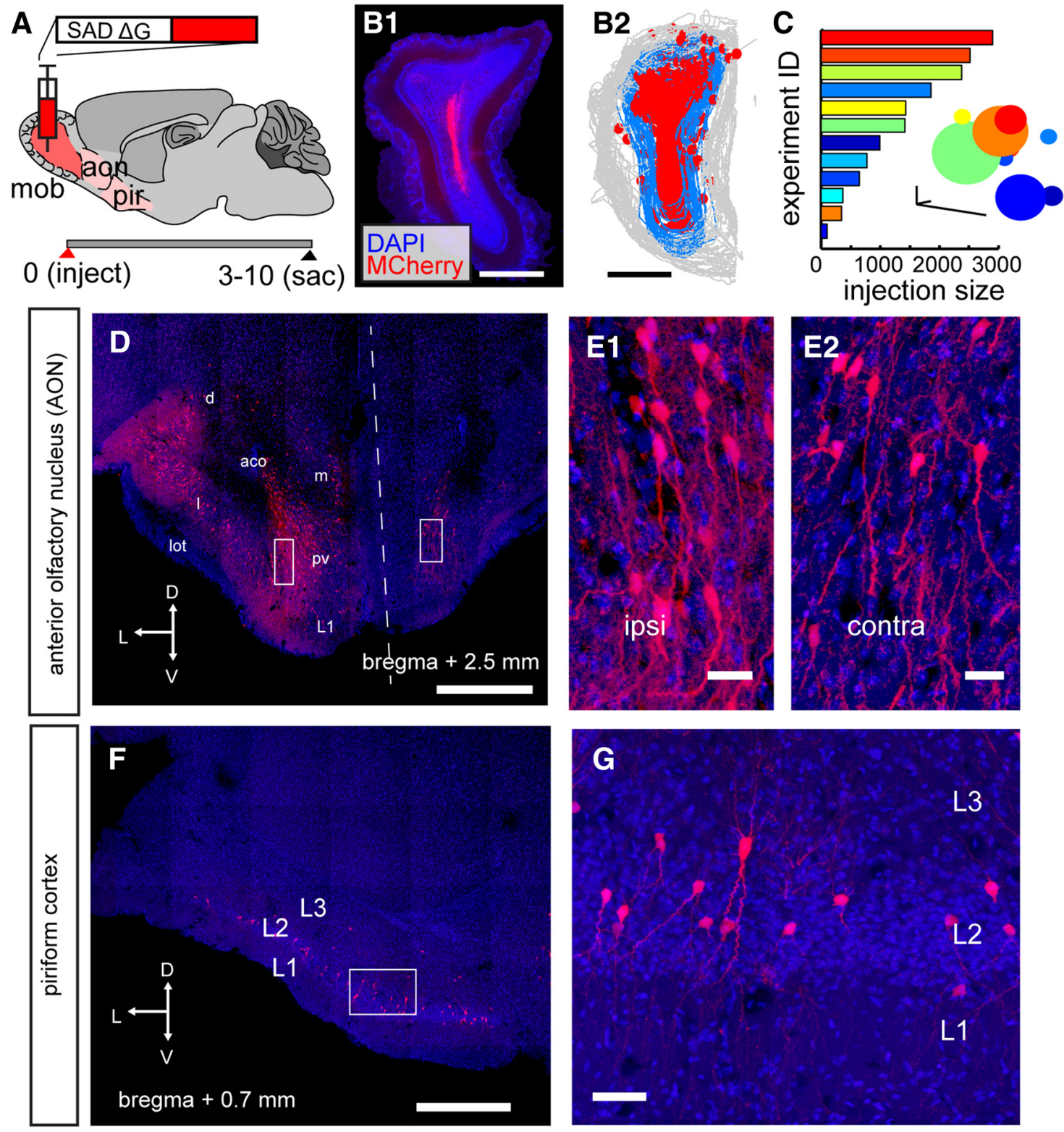

Figure 1. Retrograde label of feedback to the MOB (MOB). A, Schematic outlining experimental design. B1, Neurons labeled with mCherry rabies (red) and DAPI nuclear counterstain (blue). B2, $3 \mathrm{D}$ reconstruction of injection site in the olfactory bulb. Gray indicates the tissue edge; blue, $\mathrm{GCL}$; red, $\mathrm{mCherry}$-positive neurons. Scale bar, $500 \mu \mathrm{m}$. C, Variability in injection size across experiments $(\boldsymbol{C}$, insert) and variability in injection location; each color corresponds to an experiment. Sphere center is the location of the injection center in the GCL. The sphere radius is the injection size. Scale bar, 1 A.U. D, AON with areas of retrogradely labeled neurons in red. d, Dorsal; I, lateral; lot, lateral olfactory tract; L1, layer 1 of AON; pv, posteroventral; $m$, medial; aoc, anterior commissure. Retrogradely labeled AON neurons from ipsilateral. Scale bar, $500 \mu \mathrm{m}$. E1, E2, AON (E1) and contralateral AON (E2) from D. Scale bar, $25 \mu \mathrm{m}$. $\boldsymbol{F}$, Piriform cortex (blue) with retrogradely labeled neurons in red. L1, Layer 1; L2, layer 2; L3, layer 3; Pir, piriform cortex. Scale bar, $500 \mu \mathrm{m}$. G, Morphology of layer 2 piriform neurons. Scale bar, $50 \mu \mathrm{m}$.

site in the bulb and to ensure that we were studying feedback to the GCL, we first assessed the ratio of labeled cells within the GCL (Fig. 2D, red points within dark gray boundaries) to the number of cells outside the GCL but still within the bulb (Fig. 2D, red points outside the dark gray boundary (GCL) but within the light gray boundary, bulb tissue outline). In this example, the majority of cells (Fig. 2D, right, arrow) at the injection site were located within the GCL $(84 \%)$. Across all experiments, $80 \pm 19 \%(n=9)$ of fluorescently labeled cells were within the GCL.

We next applied our algorithm to individual coronal sections throughout the mouse brain in regions corresponding to the AON (Fig. 2E) and the piriform cortex (Fig. 2F). Our automated approach succeeded in identifying the edges of tissues in the coronal section containing the AON (Fig. 2E1,E2) and the piriform cortex (Fig. 2F1,F2) and was able to identify individually labeled neurons (Fig. 2E3,F3). From these individual examples, a 3D rendering of the mouse brain was made and retrogradely labeled neurons in the ipsilateral and contralateral $\mathrm{AON}$ and the piriform cortex were mapped into this representation (Fig. 2G). This 3D representation afforded us the ability to investigate the spatial organization of centrifugally projecting neurons within different brain regions and structures.

Asymmetric feedback projections from the AON across the two hemispheres

Although the AON is one of the major targets of M/T cell axons and the largest source of centrifugal fibers back to the bulb, comparatively little is known about its structure and function. The 

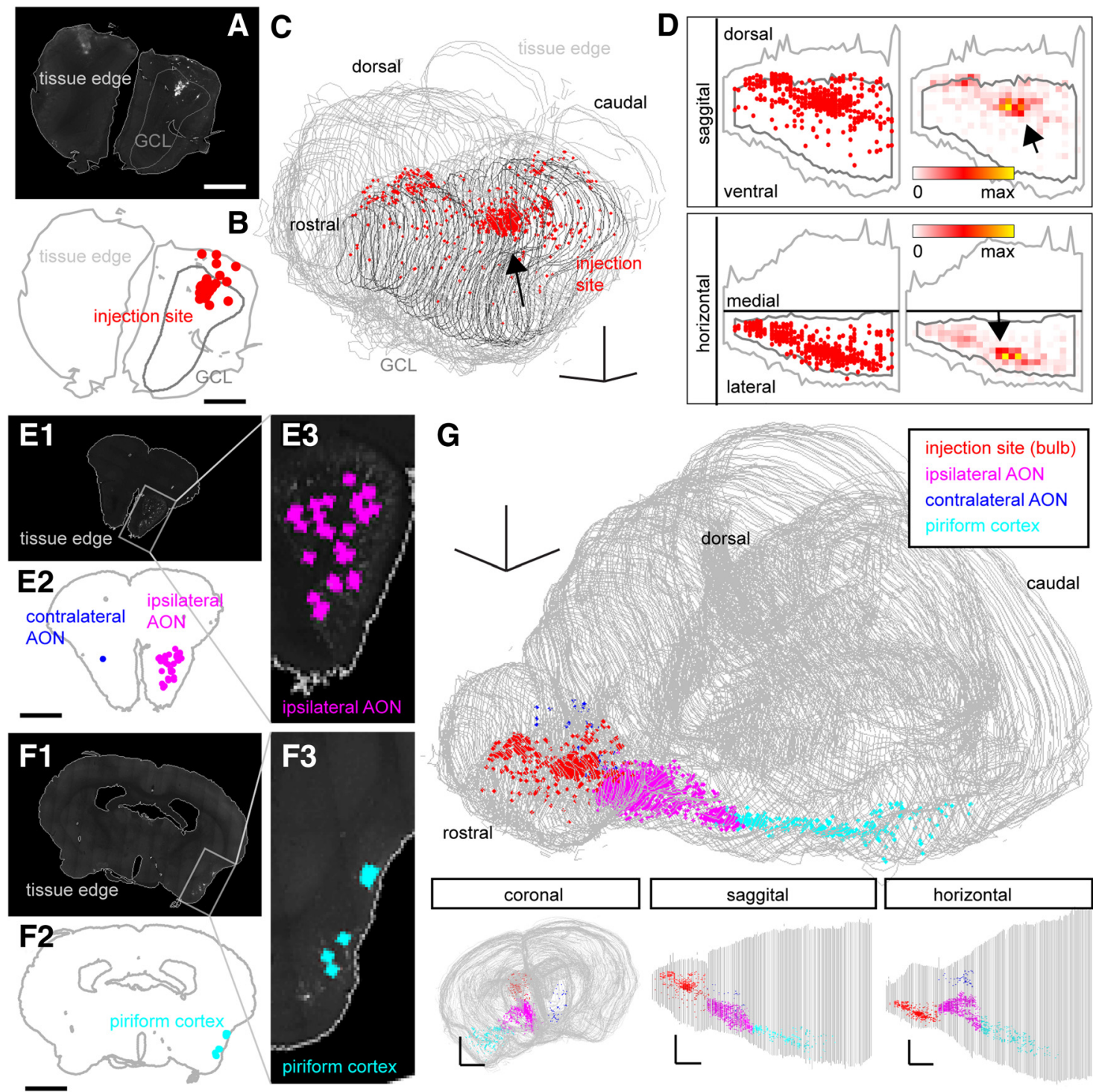

Figure 2. 3D reconstruction of the mouse brain. $A$, Example injection targeting $\mathrm{GCL}$ of the MOB. Scale bar, $500 \mu \mathrm{m}$. $\boldsymbol{B}$, Reconstruction of tissue (light gray), $\mathrm{GCL}$ boundary (dark gray), and fluorescently labeled neurons (red) from injection in $A$. Scale bar, $500 \mu \mathrm{m}$. C, 3D reconstruction of MOB (light gray), GCL boundary (dark gray), injection site (black arrow, red = labeled neurons), and retrogradely labeled cells in the bulb (red). Scale bar, $500 \mu \mathrm{m}$. D, Top left, Sagittal view of bulb in C with border of GCL (dark gray), tissue edge (light gray), and labeled neurons (red). D, Top right, Density plot of injection site (black arrow) and retrogradely labeled neurons within the bulb. Bottom left, Horizontal view of bulb from Cand (bottom, right) density plot of retrogradely labeled shows that injection is confined entirely to the ipsilateral hemisphere of the within the GCL. E1, E2, Coronal section of AON with tissue edge outlined in gray, retrogradely labeled neurons in the ipsilateral hemisphere identified in magenta, and neurons labeled in the contralateral hemisphere identified in blue from the injection site in $\boldsymbol{B}$. Scale bar, $500 \mu \mathrm{m}$. E3, Enlargement of identified neurons (magenta) reveals accuracy of automated cell-finding algorithm. $\boldsymbol{F 1}, \boldsymbol{F 2}$, Retrogradely labeled neurons in the piriform cortex (cyan) and tissue edge (gray) from the injection site in $\boldsymbol{B}$. Scale bar, 500 $\mu \mathrm{m} . \mathbf{F 3}$, Enlargement of region illustrates the accuracy of the automated cell finder across different brain regions. $\mathbf{G}, 3 \mathrm{D}$ reconstruction of whole mouse brain with retrogradely labeled neurons from the injection site, ipsilateral and contralateral AON, and the piriform cortex identified. Gray lines correspond to the edge of the tissue for each coronal section in the reconstruction. Scale bar, $500 \mu \mathrm{m}$.

AON can be subdivided into the pars externa (AONpE) and the pars principalis (AONpP). The latter is further segmented into a dorsal, ventral, medial, and lateral region (Brunjes et al., 2005). AONpE feedback to the bulb has been studied previously; axons from AONpE neurons target mirror symmetric glomeruli in the bulbs of both hemispheres, creating a bilateral olfactory map (Yan et al., 2008). In contrast, although projections from AONpP are known to target the GCL (Shipley and Adamek, 1984; Markopoulos et al., 2012), it is unclear whether these projections are organized as mirror-symmetric maps in the same way as AONpE. To address this, we studied the distribution of neurons retrogradely labeled in both the ipsilateral and contralateral AON (Fig. $3 A-E$ ) from injections in the GCL (Fig. $3 A$, inset). The boundaries of both AON hemispheres (Fig. $3 B$ ) were identified manually and mapped into a 3D representation of the brain (Fig. 3B). Dark gray lines correspond to the $\mathrm{AON}$ and light gray lines correspond to the tissue edges in each section. Retrogradely labeled neurons (Fig. 3C) were identified as blue points using the methods described in Figure 2 (Fig. 3D) and mapped into the 3D representation of the AON. First, the number of retrogradely labeled neurons in both the ipsilateral and contralateral AON correlated with the size of the injection site (Fig. $3 F$, ipsilateral AON, blue, $p=0.01, r=0.5$; contralateral AON, light blue, $p<0.005, r=$ 0.69 ) with the number of labeled neurons ranging from 38 cells to 10604 cells in the ipsilateral AON and 3 cells to 742 cells in the contralateral AON. Despite the variability across experiments, the number of labeled neurons in the ipsilateral AON also correlated with the number of labeled neurons in the contralateral 

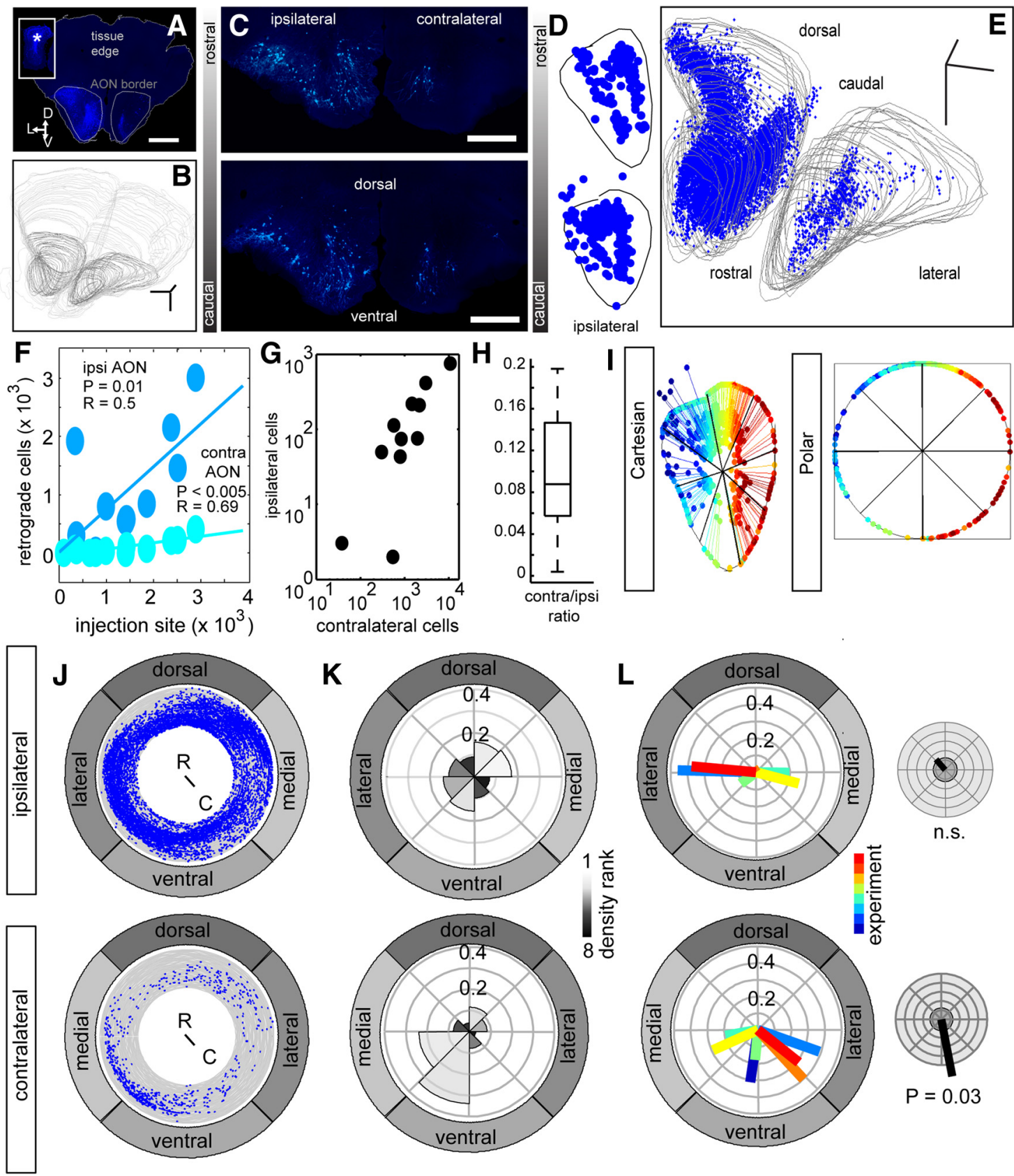

Figure 3. Organization of retrogradely labeled neurons in the AON. $A$, Retrogradely labeled neurons in the AON after injection rabies in the GCL (insert, Ai). Asterisk corresponds to the injection site. $\boldsymbol{B}, 3 \mathrm{D}$ reconstruction of tissue edge (AON and overlying cortical structures) in light gray and AON border in dark gray. $\boldsymbol{C}$, Retrogradely labeled neurons in coronal $\mathrm{AON}$ sections. $\boldsymbol{D}$, Automated cell finder identifies ipsilateral retrogradely labeled neurons in two adjacent coronal sections (blue). $\boldsymbol{E}$, Enlargement of AON from $\boldsymbol{B}$ with retrogradely labeled cells in blue. Scale bar, $500 \mu \mathrm{m} . \boldsymbol{F}$, Correlation of number of retrogradely labeled neurons in the ipsilateral (dark blue) and contralateral (light blue) hemisphere to labeled neurons in the injection site. $\boldsymbol{G}, \boldsymbol{H}$, Relationship between the number of cells in the ipsilateral and contralateral hemisphere $(\boldsymbol{G})$ and the ratio of the cell density $(\boldsymbol{H})$ in the AON. $\boldsymbol{I}$, Remapping of retrogradely labeled cells into a circular coordinate space. Colors correspond to position in a circular coordinate space. J, All remapped coronal sections for ipsilateral AON (top) and contralateral AON (bottom). Each concentric gray circle is one coronal section of AON; each blue point is one neuron. Ring spacing indicates $100 \mu \mathrm{m}$ between coronal sections. $\boldsymbol{K}$, Histogram of radial distribution of retrogradely labeled neurons from $\boldsymbol{E}$. Lightest bins correspond to the highest density. $L$, Vector representation of mean circular histogram from eight experiments (color) for ipsilateral (top) and contralateral (bottom) AON. Mean vector of retrograde neurons across eight animals (right) is shown. Error bars indicate SD.

AON (Fig. $2 G, p=0.94$ ) with a slope of 0.07 . Consistent with this, the ratio of ipsilateral to contralateral cells was $9.8 \pm 5.9 \%$ (Fig. $3 H, n=11$ ), suggesting a tight coupling of the number of cells projecting to the GCL from the two AON hemispheres. To better understand the spatial arrangement of neurons in the AON, the distribution of labeled neurons in a Cartesian coordinate space was remapped into a polar coordinate space (Fig. 3I). The position of each cell in a single coronal section was assigned a polar value ( $0-2 \pi$; Fig. $3 G$, blue dots) and placed on a circle (Fig. 3J, gray circle). The rostral-caudal axis corresponded to circles with increasingly large radii, allowing us to visualize the 3D Euclidean distribution of neurons in a 2D polar space. When we ranked the density of labeled cells based on the octants (Fig. $3 K$, highest density $=$ light, lowest density $=$ dark), fluorescently labeled neurons in the ipsilateral AON were uniformly distributed throughout all regions of the AONpP. In contrast, fluorescently 
labeled neurons from the ventral $\mathrm{AONpP}$ of the contralateral hemisphere represented a larger fraction of interhemispheric centrifugal projections (Fig. $3 L$ ). Across all experiments in which the mean position of retrogradely labeled cells was represented as a vector (Fig. $3 \mathrm{~L}$, color corresponds to individual experiment), we found that the distribution of retrogradely neurons from the ipsilateral AON was not significantly different from uniform (Fig. $3 M$, left, $p=0.85$, Raleigh test, $n=8$ ), but the distribution of retrogradely labeled cells in the contralateral AON showed a significant ventral bias (Fig. $3 M$, right, $p=0.03$, Raleigh test, $n=8$ ). Collectively, these data suggest that feedback projections from the AONpP are organized in different ways than what has been reported for $\mathrm{AONpE}$ and feedforward projections to the AON from the bulb.

\section{Retrogradely labeled piriform cortical neurons reveal principles of centrifugal fiber organization}

In addition to the AON, the piriform/olfactory cortex is both a major recipient of input from the $\mathrm{M} / \mathrm{T}$ cells of the bulb and a major source of centrifugal projections to GCs. The piriform cortex is often divided into the anterior piriform and the posterior piriform, each structurally (Shipley and Adamek, 1984; Sosulski et al., 2011) and functionally different (Choi et al., 2011; Zelano et al., 2011). Although a great deal is known about the organization of inputs to piriform cortex from the olfactory bulb (Miyamichi et al., 2011; Ghosh et al., 2011; Sosulski et al., 2011), less is known about the organization of centrifugal projections from piriform cortex back to the bulb. First, we found that retrogradely labeled cells could be seen along the total length of the rostral to caudal axis (Fig. 4A, gray line corresponds to layer 2). Over different injection sizes in the GCL, the number of retrogradely labeled neurons in the prifirom cortex correlated with the number of cells in the injection site (Fig. $4 B, p=0.016, r=0.45$ ). Furthermore, in this example (Fig. $4 C$, top) and across the population (Fig. $4 C$, bottom), the number of cells decreased in progressively more caudal sections $(p<0.001, n=10, r=0.9$, linear regression) As a result, the anterior piriform contained significantly more feedback-projecting neurons compared with those from posterior piriform ( $p<0.001$, ANOVA, $n=10)$. These results further suggest that the anterior and posterior piriform cortex are structurally distinct based on connectivity (Price and Powell, 1970; Sosulski et al., 2011) and lend evidence to the growing notion that the computations performed by the anterior and posterior piriform cortex may be functionally distinct.

Next, we investigated whether there was finer structure in the spatial arrangement of cells that projected to the GCL. To do this, we first examined the laminar organization of feedbackprojecting neurons. A curve was manually defined corresponding to the border along layer 2 of the piriform cortex and the distance of each individual retrogradely labeled neuron orthogonal to that curve was calculated as a measure of laminar position (Fig. 4D). We found that the normalized distribution of neuronal positions of retrogradely labeled cells was significantly different from a Gaussian centered at the layer 2 boundary ( $p<0.005$, ANOVA).

In addition to structure across the laminae of the piriform cortex, we wished to explore other features of the spatial organization within the area. To do this, the position of retrogrogradely labeled neurons was remapped onto a $2 \mathrm{D}$ space by projecting the location of each neuron in the coronal section (Fig. $4 E$, light red) onto an arc (Fig. $4 E$, dark red points) defined by the cell-dense band that constitutes layer 2 (Fig. $4 E$, gray line). This remapping preserved nearest neighbor distances $\left(R^{2}=0.97\right.$, Fig. $\left.4 F\right)$, ensuring that any topological transformations did not affect statistical features of the relative positions of retrogradely labeled cells to one another. Individual coronal representations were aligned and visualized as a $2 \mathrm{D}$ plane (Fig. $4 G$, top) in which each gray line corresponded to a single coronal section. Clusters of neurons could be observed throughout the $2 \mathrm{D}$ representation (blue arrows), with peaks in the clustering revealed in a density map (Fig. $4 G$, bottom). To evaluate the statistics of this arrangement, we developed a method of generating neurons that were randomly distributed throughout the piriform cortex. Briefly, randomly distributed cells were generated by drawing from positions of layer $2(R)$ in each coronal section matched to the original data for each experiment. $X-Y$ positions were drawn from of a distribution of all possible cell locations $(n)$ along the curve defined by layer 2 in each coronal section. As a result, random feedback maps could be generated for each experiment and compared with the distribution of retrogradely labeled neurons in that same experiment. From this, the mean pairwise distances between retrogradely labeled cells in the real data (Fig. $4 H$, black, mean distance $=119 \pm 91 \mu \mathrm{m}$ ) to those of a random distribution (Fig. $4 H$, gray, mean distance $239 \pm 157 \mu \mathrm{m}$ ) were determined. In this single experiment (Fig. $4 H, p<0.001$, ANOVA) and across all experiments (Fig. $4 I, p<0.01$, paired $t$ test), we found a significant difference between the mean distance between nearest neighbors in the real data and that of the random distribution. Therefore, feedback-projecting cells were closer together than would be expected if centrifugally projecting neurons were spatially random. We note that, when pairwise distances were plotted as a function of the injection size (Fig. 4J), we found no significant correlation for either the data or the random distributions ( $p=0.62$ data, $p=0.32$ random). A number of factors could account for this. First, neither injection size nor the intercell distances were distributed normally, so correlations between mean values may not exist. Second, injection size may be related to other properties of clustering, such as the number of clusters or the intercluster spacing, none of which may be captured by the intercell distance differences.

To address these issues and to further explore this spatial clustering, we made injections of two different fluorophores (GFP and BFP, $n=3$ ), each expressed in a g-deleted rabies virus, into nearby regions of the GCL in the same animal (Fig. $4 K$, inset). Centrifugally projecting neurons were retrogradely labeled throughout the mouse brain, including in the piriform cortex (Fig. $4 K$ ). These cells were then mapped into a $3 \mathrm{D}$ representation (Fig. $4 K$ ) and, consistent with our previous findings, retrogradely labeled cells were the most dense in the anterior piriform cortex for both BFP (Fig. 4L) and GFP (Fig. 4M) injections. Spatially distinct patches became apparent when a difference image was made of the two distributions (Fig. $4 n$, arrows), further suggesting that feedback-projecting neurons in the piriform cortex were organized in nonrandom patterns.

A number of features can affect the appearance of clustered patterns in these experiments, including differences in the sizes of the injections (Fig. 1B), the location of the different injections (Fig. 1C), and differences in the viral titers/infectivity. In addition, intrinsic biases pertaining to the infectivity of different viruses (e.g., BFP vs GFP) could also affect the spatial distribution of retrogradely labeled neurons, not necessarily in a random way. Each of these differences could generate the appearance of clusters of retrogradely labeled cells within the piriform cortex that would be due to experimental differences rather than underlying features of the connectivity from the piriform cortex to the bulb. To exclude these sources of error, we analyzed the statistics of the clusters across different experiments (different injection size, dif- 
A dorsal

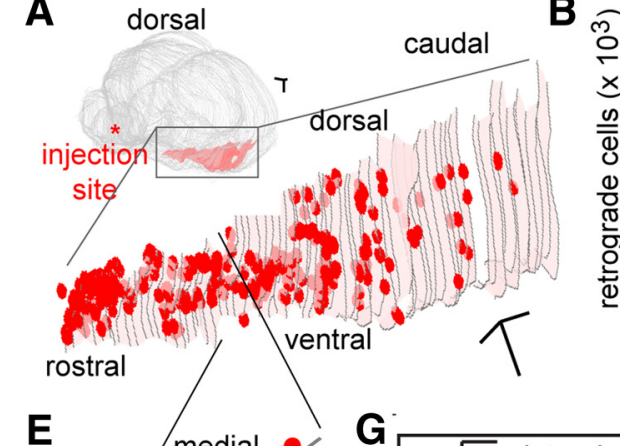

E

layer 2
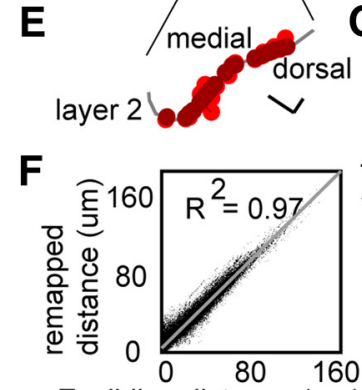

Euclidian distance (um)

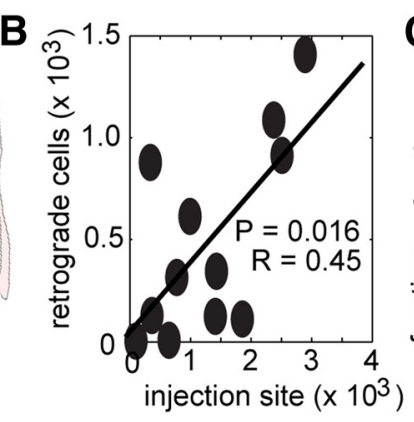

C

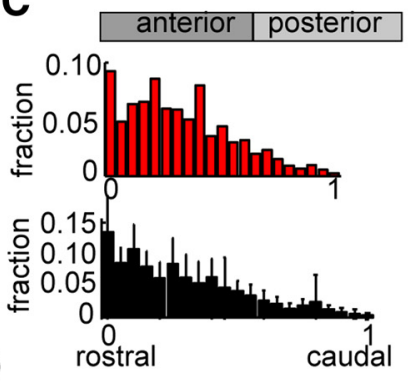

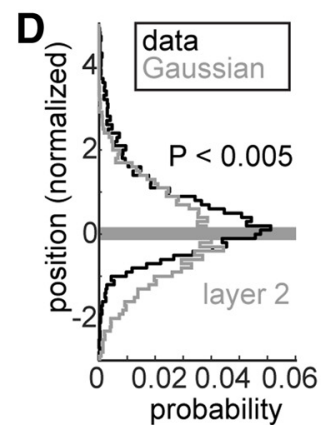

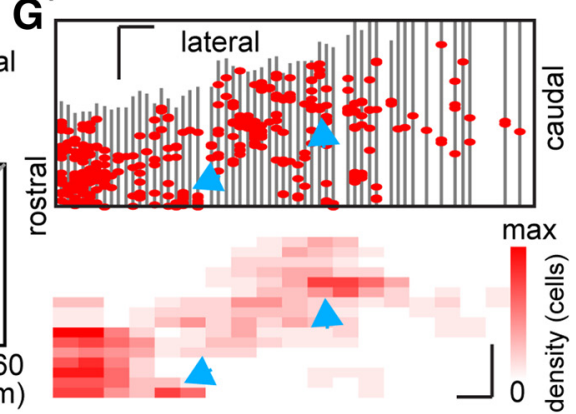

H
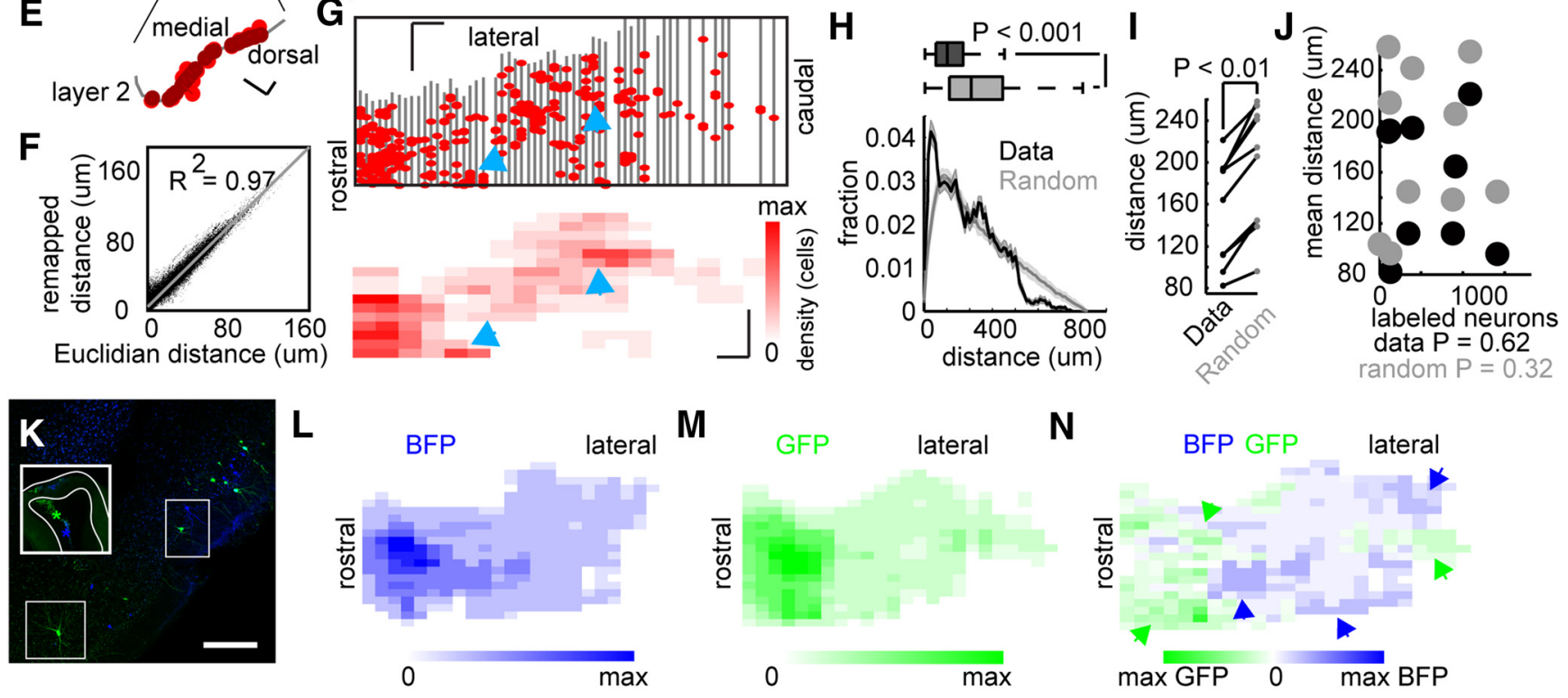

$\mathbf{N}$
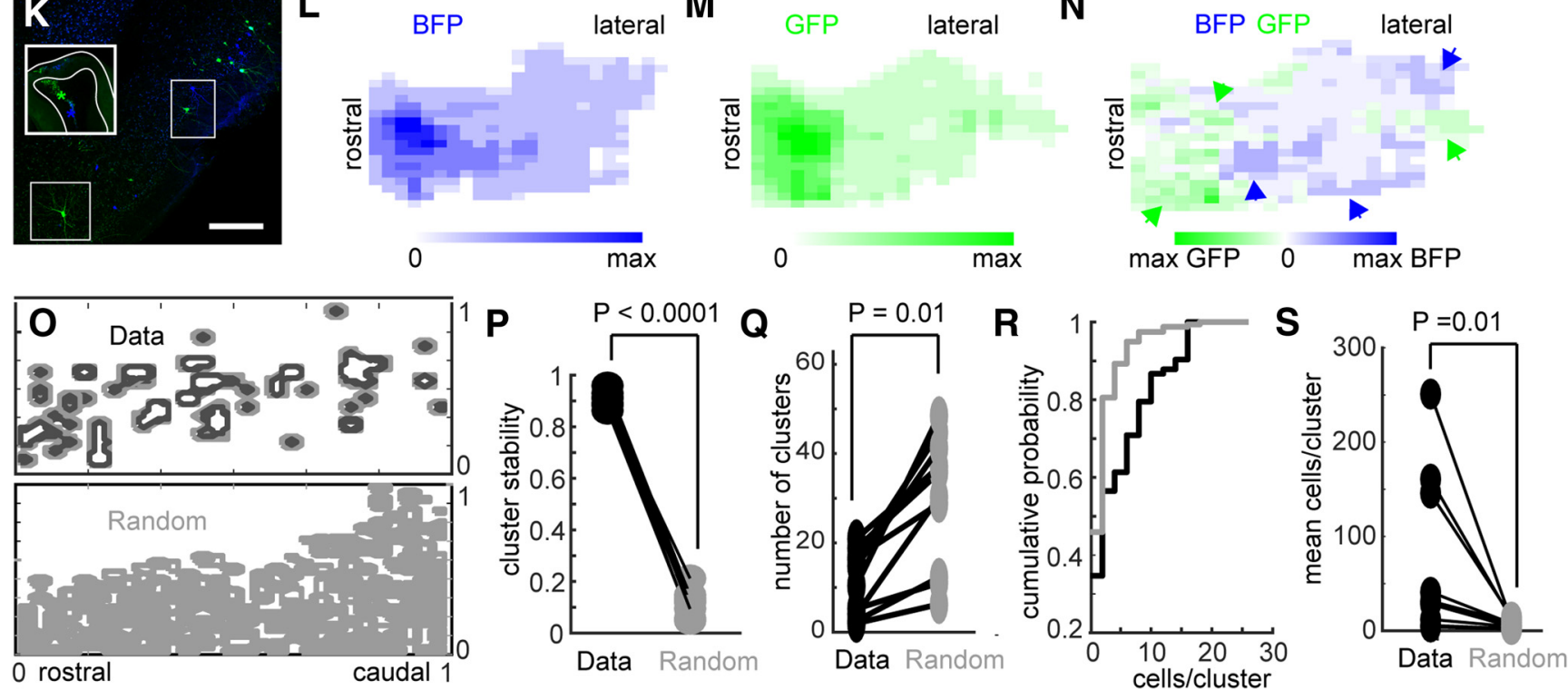

Figure 4. Organization of retrogradely labeled neurons in the piriform cortex. $A$, Retrogradely labeled cells in the piriform cortex (red) mapped onto a whole mouse brain reconstruction from a g-deleted rabies injection in the GCL. $\boldsymbol{A}$, Enlargement, Gray lines corresponding to layer 2 from each coronal section. $\boldsymbol{B}$, Correlation of injection size in GCL to number of labeled neurons in piriform cortex. C, Top, Rostral-caudal distribution of retrogradely labeled cells from A. C, Bottom, Mean rostral-caudal distribution of retrogradely labeled cells across all experiments. Error bars indicate SD. D, Laminar distribution of retrogradely labeled neurons in piriform cortex (black) compared with a Gaussian distribution centered at the layer 2 border. $\boldsymbol{E}$, Projection (new neuron position $=$ dark red dots, projection $=$ red lines) of retrogradely labeled neurons (light red) onto a line (gray) through layer 2 . This projection is then warped to a linear representation. $\boldsymbol{F}$, Correlation of pairwise distances in the Euclidean space to the pairwise distances in the remapping demonstrate that local spatial relationships are preserved during remapping. G, Top, Remapping of retrogradely labeled cells in piriform to a 2D surface. G, Bottom, Density plot of retrogradely labeled neurons. Scale bar, $500 \mu \mathrm{m}$. $\boldsymbol{H}$, Histogram of mean pairwise distances between neurons (black) from retrograde data and pairwise distances from randomly distributed cells (gray). Population means for the example (top) are shown. Error bar indicates SD. $I$, Mean pairwise distances from experiments (black) versus from random distributions (gray). J, Mean pairwise distance is not correlated with the number of retrogradely labeled neurons for either data (black, $p=0.62)$ or random data (gray, $p=0.32$ ) in the piriform cortex. $\boldsymbol{K}$, Injection of two g-deleted rabies virus constructs into the GCL (insert) and retrograde label of neurons in piriform cortex. Scale bar, $500 \mu \mathrm{m}$. $\boldsymbol{L}-\boldsymbol{N}$, Density of retrogradely labeled BFP $(\boldsymbol{L})$ and GFP $(\boldsymbol{M})$ neurons and difference in density distributions $(\boldsymbol{N})$. Arrows correspond to clusters of feedback projecting neurons. $\mathbf{0}$, Top, Outline of clusters from multiple resamples of a single experiment. $\mathbf{O}$, Bottom, Outline of clusters from multiple resamples of random data for the same experiment as in $\mathbf{O}$ (top). $\boldsymbol{P}$, Cluster stability as measured by correlation of cluster shape from resample to resample. $\boldsymbol{Q}$, Number of clusters per experiment calculated from the data (black) compared with the random data (gray). $\boldsymbol{R}$, Cumulative histogram of number of cells per cluster in the experimental data (black) compared with random data (gray). S, Mean number of cells per cluster across all experiments (black) compared with random data (gray).

ferent location, different generic fluorescent protein (XFP), etc.) using an automated algorithm that quantified the structure of clustered patches as a function of different experimental parameters. The spatial density maps (Fig. $4 G, L-N$ ) of retrogradely labeled cells were used to generate cluster boundaries (defined as 1 SD greater than the mean cell density) for both data from each experiment (Fig. 4O, top) and from randomly generated distributions within the same piriform cortex (Fig. $4 O$, bottom). Re- 
peated resampling of these maps, done through bootstapping from either the experimental data or from random distributions across the piriform cortex, allowed for quantification of cluster structure both within a single experiment and across experiments. Although repeated resamples of retrogradely labeled cells from the experimental data consistently resulted in stable patterns of clusters (Fig. 4O, top), cluster maps varied from resample to resample in the random distributions (Fig. 4O, bottom). The stability of these patterns was quantified using a cluster stability index that calculated the correlation coefficient of cluster shapes from resample to resample in both the data and random maps. Clusters in the real data were stable (Fig. $4 P$, cluster correlation $=$ $0.9 \pm 0.3, n=100$ resamples each for 10 experiments) but clusters varied dramatically from resample to resample in the random data (Fig. $4 P$, cluster correlation $=0.12 \pm 0.5, n=100$ resamples each for 10 experiments). This difference was statistically significant (Fig. $4 P, p<0.0001, n=10$ data, $n=10$ random, 10 injections, Wilcoxon rank-sum). Furthermore, because clusters were stable across experiments (Fig. $4 P$ ) of different injection size (Fig. $4 B$ ) and different XFPs, clustering was unlikely the result of experimental variability.

Because injection size and location varied from experiment to experiment, so too did the mean number of clusters observed, varying from 2 to 21 in the experimental data and from 5 to 53 in the random data. Across all experiments, we found that there were $11.4 \pm 7.5$ clusters on average in the experimental data and $29.9 \pm 15.2$ clusters in the random data, a difference that was statically significant (Fig. $4 Q, n=10, p=0.01$, Wilcoxon ranksum). In addition to differences in the number of clusters observed experiments, the number of cells per cluster across different experiments was highly variable. Despite the interexperimental differences, the number of cells per cluster in any given experiment was greater in the real data compared with the randomly generated clusters for the same experiment (Fig. 4R). Consistent with this, across all experiments, we found $71 \pm 84$ cells per cluster (Fig. $4 S, n=10$ experiments) in the real data compared with $7 \pm 4$ cells per cluster in the random data (Fig. $4 S$, $n=10$ ), a difference that was statistically significant (Fig. $4 S, p=$ 0.01, Wilcoxon rank-sum).

These data suggest that feedback-projecting cells in the piriform cortex are spatially organized and that this organization may be different from the pattern of innervation of feedforward input from the bulb that has been described previously.

\section{Discussion}

By combining a retrograde viral labeling strategy with wholebrain 3D reconstructions (Padmanabhan et al., 2010), we describe the structure of centrifugal projections to the GCL of the bulb from both the AON and the piriform cortex, the two major targets of information from the MOB and the two largest sources of feedback to the bulb. We first confirmed the validity of our method, identifying known projections from olfactory areas such as the $\mathrm{AON}, \mathrm{nLOT}$, and piriform cortex and neuromodulatory areas such as the HDB back to the GCL of the bulb. We identified a number of features of the spatial organization of projecting neurons from the AON and the piriform cortex that might provide insight into olfactory coding and ultimately olfactory behavior.

\section{Dorsal-ventral differences in feedback from the AON to the bulb disrupt principles of feedforward organization}

Anatomical distinctions based on space (dorsal vs ventral) are often essential for understanding how information is processed in sensory circuits, including the olfactory system. Projections from dorsal and ventral $\mathrm{M} / \mathrm{T}$ cells target dorsal and ventral regions of the AON, respectively (Miyamichi et al., 2011), thereby preserving a dorsal/ventral segregation of olfactory information. This organization is thought to have important consequences for separating circuits into those that guide innate (dorsal) versus learned (ventral) behaviors (Kobayakawa et al., 2007). Interestingly, this dorsal-ventral organization is preserved in the projections from AONpE back to the bulb (Yan et al., 2008). In contrast, we found that centrifugal projections from the ventral region of the contralateral AONpP constituted the majority of interhemispheric feedback projections to the GCL, suggesting an important property of AON feedback. First, early experiments in rats (Kucharski and Hall, 1987) and more recent work in humans (Mainland et al., 2002) has demonstrated that olfactory information learned in one hemisphere can be relayed to the other. Feedforward circuits in mammals from the bulb to cortical processing centers are almost exclusively ipsilateral, suggesting that as interhemispheric integration of olfactory information is done, one possibility pathway is feedback. The overrepresentation of feedback from the ventral AON to the bulb may be one anatomical way in which sharing of learned olfactory information is facilitated between hemispheres.

\section{Feedback from piriform cortex to the GCL is spatially clustered}

Distributed feedforward projections from the bulb to the olfactory cortex (Ghosh et al., 2011; Miyamichi et al., 2011; Sosulski et al., 2011) result in neighboring piriform neurons that are randomly tuned to different odor combinations (Stettler and Axel, 2009). In contrast, we found patches of nearby neurons that projected to similar regions of the GCL corresponding to our injection site. We hypothesize that the proximity of centrifugally projecting neurons to each another allows them to interact through local inhibitory connections (Poo and Isaacson, 2009; Suzuki and Bekkers, 2010). By synthesizing the activity of local ensembles of neurons (Zelano et al., 2011), clustered feedback may further sparsen incoming odor representations in the MOB (Otazu et al., 2015). In addition, recent theoretical work has shown that clustered connectivity can give rise to complex temporal patterns of activity on long time scales (Litwin-Kumar and Doiron, 2012). Temporally rich activity from centrifugal projections could thus be layered onto the existing dynamics of neuron firing (Friedrich and Laurent, 2001; Kapoor and Urban, 2006; Wesson et al., 2009; Padmanabhan and Urban, 2014) generated by local circuits in the bulb (Friedrich and Laurent, 2001; Laurent, 2002).

Although our work reveals previously unknown organizational principles of feedback anatomy, it does not address important properties of those maps, including their relationship and connectivity to ongoing adult neurogenesis in the MOB (Mouret et al., 2009; Lazarini and Lledo, 2011; Deshpande et al., 2013). As newborn granule cells, which are the primary targets of these feedback projections are turned over throughout the organism's life, including as a result of changes in experience (Lin et al., 2010), these maps may be further affected by changes in the circuit. In addition, this work does not explore how these circuits are formed over the course of development (Brunjes et al., 2014). These questions go beyond the scope of this work and may be the subject of future investigations.

Sensory maps in the mammalian brain, in which similar properties of sensory stimuli (retinotopy, tonotopy, etc.) are represented by neurons adjacent to one another, reflect a fundamental 
organizing feature of the neocortex. Olfaction, however, is distinct in this regard because no single or simple combination of chemical features (carbon chain length, functional group, etc.) appears to have a continuous map in the cortex (Murthy, 2011) such as, for example, the orientation columns in primary visual cortex (Hubel and Wiesel, 1968; Bonhoeffer and Grinvald, 1991).

In this study, we described the structure of feedback projections from cortical areas back to the inhibitory neurons in the MOB. As in the case of feedforward projections from the M/T cells of the bulb to cortical targets (Yan et al., 2008; Soucy et al., 2009; Ghosh et al., 2011; Miyamichi et al., 2011; Sosulski et al., 2011), the organization of centrifugal projections varied from brain area to brain area. We hypothesize that, similar to the feedforward connectivity, the differential organization of centrifugal feedback projections from cortical areas back to the bulb may be important for guiding olfactory behavior (Kobayakawa et al., 2007; Katz et al., 2008; Root et al., 2014). Importantly, these differences are not simply recapitulations of feedforward structure. Instead, feedback projections are organizationally distinct. As a result, any putative maps in the olfactory system, be they the ones that originate from the AON and the piriform cortex as we have studied, or projections more generally for diverse areas such as neuromodulatory centers (Rothermel et al., 2014), may be as much about the behaviorally relevant outputs in response to odors as they are about the chemical structure of those odors.

\section{References}

Ahmed B, Anderson JC, Douglas RJ, Martin KA, Nelson JC (1994) Polyneuronal innervation of spiny stellate neurons in cat visual cortex. J Comp Neurol 341:39-49. CrossRef Medline

Babadi B, Sompolinsky H (2014) Sparseness and expansion in sensory representations. Neuron 83:1213-1226. CrossRef Medline

Bonhoeffer T, Grinvald A (1991) Iso-orientation domains in cat visual cortex are arranged in pinwheel-like patterns. Nature 353:429-431. CrossRef Medline

Boyd AM, Sturgill JF, Poo C, Isaacson JS (2012) Cortical feedback control of olfactory bulb circuits. Neuron 76:1161-1174. CrossRef Medline

Boyd AM, Kato HK, Komiyama T, Isaacson JS (2015) Broadcasting of cortical activity to the olfactory bulb. Cell Rep 10:1032-1039. CrossRef Medline

Brunjes PC, Illig KR, Meyer EA (2005) A field guide to the anterior olfactory nucleus (cortex). Brain Res Brain Res Rev 50:305-335. CrossRef Medline

Brunjes PC, Collins LN, Osterberg SK, Phillips AM (2014) The mouse olfactory peduncle. 3. Development of neurons, glia, and centrifugal afferents. Front Neuroanat 8:44. CrossRef Medline

Callaway EM, Luo L (2015) Monosynaptic circuit tracing with glycoproteindeleted rabies viruses. J Neurosci 35:8979-8985. CrossRef Medline

Choi GB, Stettler DD, Kallman BR, Bhaskar ST, Fleischmann A, Axel R (2011) Driving opposing behaviors with ensembles of piriform neurons. Cell 146:1004-1015. CrossRef Medline

Davison IG, Ehlers MD (2011) Neural circuit mechanisms for pattern detection and feature combination in olfactory cortex. Neuron 70:82-94. CrossRef Medline

Deshpande A, Bergami M, Ghanem A, Conzelmann KK, Lepier A, Götz M, Berninger B (2013) Retrograde monosynaptic tracing reveals the temporal evolution of inputs onto new neurons in the adult dentate gyrus and olfactory bulb. Proc Natl Acad Sci U S A 110:E1152-E1161. CrossRef Medline

Friedrich RW, Laurent G (2001) Dynamic optimization of odor representations by slow temporal patterning of mitral cell activity. Science 291:889894. CrossRef Medline

Ghosh S, Larson SD, Hefzi H, Marnoy Z, Cutforth T, Dokka K, Baldwin KK (2011) Sensory maps in the olfactory cortex defined by long-range viral tracing of single neurons. Nature 472:217-220. CrossRef Medline

Gonzalez RC, Woods RE, Eddins SL (2004) Digital image processing using MATLAB, 1st ed. Upper Saddle River, NJ: Pearson Prentice Hall.

Haberly LB, Price JL (1978) Association and commissural fiber systems of the olfactory cortex of the rat. J Comp Neurol 178:711-740. CrossRef Medline
Hubel DH, Wiesel TN (1968) Receptive fields and functional architecture of monkey striate cortex. J Physiol 195:215-243. CrossRef Medline

Kapoor V, Urban NN (2006) Glomerulus-specific, long-latency activity in the olfactory bulb granule cell network. J Neurosci 26:11709-11719. CrossRef Medline

Katz DB, Matsunami H, Rinberg D, Scott K, Wachowiak M, Wilson RI (2008) Receptors, circuits, and behaviors: new directions in chemical senses. J Neurosci 28:11802-11805. CrossRef Medline

Kobayakawa K, Kobayakawa R, Matsumoto H, Oka Y, Imai T, Ikawa M, Okabe M, Ikeda T, Itohara S, Kikusui T, Mori K, Sakano H (2007) Innate versus learned odour processing in the mouse olfactory bulb. Nature 450:503-508. CrossRef Medline

Kucharski D, Hall WG (1987) New routes to early memories. Science 238: 786-788. CrossRef Medline

Laurent G (2002) Olfactory network dynamics and the coding of multidimensional signals. Nat Rev Neurosci 3:884-895. CrossRef Medline

Lazarini F, Lledo PM (2011) Is adult neurogenesis essential for olfaction? Trends Neurosci 34:20-30. CrossRef Medline

Lin CW, Sim S, Ainsworth A, Okada M, Kelsch W, Lois C (2010) Genetically increased cell-intrinsic excitability enhances neuronal integration into adult brain circuits. Neuron 65:32-39. CrossRef Medline

Litwin-Kumar A, Doiron B (2012) Slow dynamics and high variability in balanced cortical networks with clustered connections. Nat Neurosci 15: 1498-1505. CrossRef Medline

Mainland JD, Bremner EA, Young $n$, Johnson BN, Khan RM, Bensafi M, Sobel $n$ (2002) Olfactory plasticity: one nostril knows what the other learns. Nature 419:802. CrossRef Medline

Markopoulos F, Rokni D, Gire DH, Murthy VN (2012) Functional properties of cortical feedback projections to the olfactory bulb. Neuron 76: 1175-1188. CrossRef Medline

Meister M, Bonhoeffer T (2001) Tuning and topography in an odor map on the rat olfactory bulb. J Neurosci 21:1351-1360. Medline

Miyamichi K, Amat F, Moussavi F, Wang C, Wickersham I, Wall NR, Taniguchi H, Tasic B, Huang ZJ, He Z, Callaway EM, Horowitz MA, Luo L (2011) Cortical representations of olfactory input by trans-synaptic tracing. Nature 472:191-196. CrossRef Medline

Mombaerts P, Wang F, Dulac C, Chao SK, Nemes A, Mendelsohn M, Edmondson J, Axel R (1996) Visualizing an olfactory sensory map. Cell 87:675-686. CrossRef Medline

Mouret A, Murray K, Lledo PM (2009) Centrifugal drive onto local inhibitory interneurons of the olfactory bulb. Ann $n$ Y Acad Sci 1170:239-254. CrossRef Medline

Murthy VN (2011) Olfactory maps in the brain. Annu Rev Neurosci 34: 233-258. CrossRef Medline

Osakada F, Callaway EM (2013) Design and generation of recombinant rabies virus vectors. Nat Protoc 8:1583-1601. CrossRef Medline

Osakada F, Mori T, Cetin AH, Marshel JH, Virgen B, Callaway EM (2011) New rabies virus variants for monitoring and manipulating activity and gene expression in defined neural circuits. Neuron 71:617-631. CrossRef Medline

Otazu GH, Chae H, Davis MB, Albeanu DF (2015) Cortical feedback decorrelates olfactory bulb output in awake mice. Neuron 86:1461-1477. CrossRef Medline

Padmanabhan K, Eddy WF, Crowley JC (2010) A novel algorithm for optimal image thresholding of biological data. J Neurosci Methods 193:380384. CrossRef Medline

Padmanabhan K, Urban NN (2014) Disrupting information coding via block of 4-AP-sensitive potassium channels. J Neurophysiol 112:10541066. CrossRef Medline

Petzold GC, Hagiwara A, Murthy VN (2009) Serotonergic modulation of odor input to the mammalian olfactory bulb. Nat Neurosci 12:784-791. CrossRef Medline

Poo C, Isaacson JS (2009) Odor representations in olfactory cortex: "sparse" coding, global inhibition, and oscillations. Neuron 62:850-861. CrossRef Medline

Price JL, Powell TP (1970) An experimental study of the origin and the course of the centrifugal fibres to the olfactory bulb in the rat. J Anat 107:215-237. Medline

Root CM, Denny CA, Hen R, Axel R (2014) The participation of cortical amygdala in innate, odour-driven behaviour. Nature 515:269-273. CrossRef Medline

Rothermel M, Carey RM, Puche A, Shipley MT, Wachowiak M (2014) Cho- 
linergic inputs from Basal forebrain add an excitatory bias to odor coding in the olfactory bulb. J Neurosci 34:4654-4664. CrossRef Medline

Saito H, Chi Q, Zhuang H, Matsunami H, Mainland JD (2009) Odor coding by a mammalian receptor repertoire. Sci Signal 2:ra9. CrossRef Medline

Shipley MT, Adamek GD (1984) The connections of the mouse olfactory bulb: a study using orthograde and retrograde transport of wheat germ agglutinin conjugated to horseradish peroxidase. Brain Res Bull 12:669688. CrossRef Medline

Sosulski DL, Bloom ML, Cutforth T, Axel R, Datta SR (2011) Distinct representations of olfactory information in different cortical centres. Nature 472:213-216. CrossRef Medline

Soucy ER, Albeanu DF, Fantana AL, Murthy VN, Meister M (2009) Precision and diversity in an odor map on the olfactory bulb. Nat Neurosci 12:210-220. CrossRef Medline

Stettler DD, Axel R (2009) Representations of odor in the piriform cortex. Neuron 63:854-864. CrossRef Medline

Suzuki $n$, Bekkers JM (2010) Inhibitory neurons in the anterior piriform cortex of the mouse: classification using molecular markers. J Comp Neurol 518:1670-1687. CrossRef Medline
Urban NN, Sakmann B (2002) Reciprocal intraglomerular excitation and intra- and interglomerular lateral inhibition between mouse olfactory bulb mitral cells. J Physiol 542:355-367. CrossRef Medline

Wesson DW, Verhagen JV, Wachowiak M (2009) Why sniff fast? The relationship between sniff frequency, odor discrimination, and receptor neuron activation in the rat. J Neurophysiol 101:1089-1102. Medline

Wickersham IR, Finke S, Conzelmann KK, Callaway EM (2007) Retrograde neuronal tracing with a deletion-mutant rabies virus. Nat Methods 4:47-49. Medline

Willhite DC, Nguyen KT, Masurkar AV, Greer CA, Shepherd GM, Chen WR (2006) Viral tracing identifies distributed columnar organization in the olfactory bulb. Proc Natl Acad Sci U S A 103:12592-12597. CrossRef Medline

Yan Z, Tan J, Qin C, Lu Y, Ding C, Luo M (2008) Precise circuitry links bilaterally symmetric olfactory maps. Neuron 58:613-624. CrossRef Medline

Zelano C, Mohanty A, Gottfried JA (2011) Olfactory predictive codes and stimulus templates in piriform cortex. Neuron 72:178-187. CrossRef Medline 